\title{
SIMULATION OF SOFT ROBUST NONLINEAR CONTROL OF A PMSM
}

\author{
Viehweider, A. \& Wittmann, B. \\ Institute of Automation and Control, Vienna University of Technology, \\ Gusshausstr. 27-29, A -1040 Vienna, Austria \\ E-mail: viehweider@acin.tuwien.ac.at
}

\begin{abstract}
A backstepping controller for a permanent magnet synchronous motor (PMSM) with a nonlinear load is devised. In order to improve the control law dynamics a technique introduced by Freeman and Kokotovic is adapted for the special application. The PMSM has been modelled with special consideration of the cross coupling effect. As compared to the conventional speed control approach higher precision in following a speed reference signal is achieved. The effect of high control law dynamics can be attenuated by proper forming of the Lyapunov function. By the use of simulation the effectiveness of the approach is demonstrated.
\end{abstract}

Key Words: Robust Backstepping, Nonlinear Control, Permanent Magnet Synchronous Motor, Cross Coupling Modelling, Soft Control Law

\section{INTRODUCTION}

Nonlinear systems are a special challenge for every control engineer. A PMSM with a constant load is a nonlinear system that can be made linear by an appropriate decoupling network. This situation changes as far as nonlinear loads are concerned. We want to devise robust control laws for a PMSM with an uncertain nonlinear load. That means that we know only upper and lower bounds of the speed-torque characterstic of the load (for example a rotary pump) but not the characteristic itself. A robust control system should cope with different kind of uncertainties of the underlying process to be controlled. The achieved precision in a speed tracking application should be enhanced by use of the introduced approach. At the same time the control law amplitude and dynamics should be contained within a reasonable range.

Backstepping is a powerful control technique for nonlinear systems in strict feedback form $[1,2]$ and the number of articles dealing with applications of this method is increasing. We refer exemplary to [3], [4] and [5]. There exist different methods to cope with uncertainty. The two main classes are adaptive methods and robust methods. As far as speed control of a PMSM is concerned different authors have developed approaches for adaptive backstepping. In [6] a feedback linearisation approach is used to partially simplify the system and to obtain the standard form for backstepping control design. Then an asymptotically stable adaptive backstepping speed controller is devised. The results following a certain speed reference of the adaptive backstepping controller are compared with the results of the pure feedback linearisation controller. [7] discusses an adaptive backstepping approach for a PMSM by neglecting the electrical properties.

Adaptive control is based on the assumption that the unknwon parameters are constant or only slowly time varying. Robust control deals with parameters which are time varying within certain bounds. The knowledge of the bounds is essential. An inherent feature of robust backstepping is its aggressive non smooth control law. In [8] an approach unifying the 
adaptive and robust technique is presented. The parameters are divided into nearly constant parameters with a wide range of variation and parameters with less deviation from its rated values. The first kind of parameters is treated with an adaptive technique and the second kind by using robust techniques. A simplification of the controller design and reduction of the control authority is obtained.

This article introduces another method: Robust backstepping with appropriate measures to counteract the high control amplitudes and high control law dynamics. Since backstepping is a Lyapunov function based control technique, special forming of the Lyapunov function leads to better (softer) control laws. The idea behind a special forming of the Lyapunov function is to relax the requirements on the Lyapunov function itself: The Lyapunov function is no longer just the sum of the square of single states (in any coordinate system). Thereby we gain a softer control law. We want to show within this article how the concept can be applied to a PMSM and are one step before the application by showing the validness of the method by simulation runs. The method guarantees high tracking precision by reasonable control authority without any adaptation of parameters. The contribution of this article is the application and adaptation of the method to the pecularities of the PMSM.

Precise modelling of the PMSM is an art of its own. As representative for other occuring effects of the PMSM we have modelled the implications of cross coupling and current dependent inductivities.

\section{MATHEMATICAL MODEL OF THE PMSM}

Fig. 1 shows the cross-section of a PMSM (N and S denotes the North- and South Poles of the permanent magnets) and the definition of the synchronous $(d, q)$ reference frame, which is the basis for the following considerations.

The per unit (p.u.) stator voltage equations of the PMSM [10],[11] in a synchronous reference frame is characterized by (1).

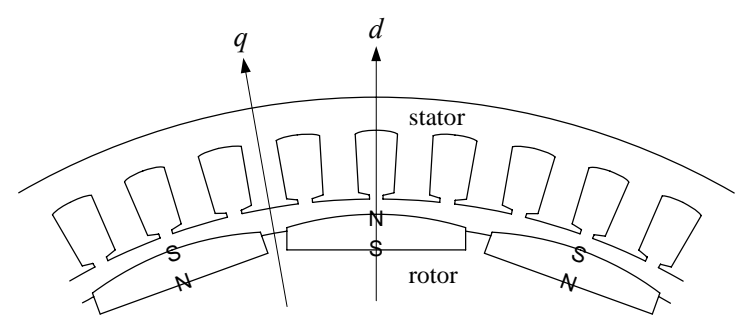

Figure 1: Cross-section of a PMSM with the definition of the synchronous $(d, q)$ reference frame.

$$
\begin{aligned}
& u_{S d}=r_{S} i_{S d}+\frac{d \psi_{S d}}{d \tau}-\omega_{m} \psi_{S q} \\
& u_{S q}=r_{S} i_{S q}+\frac{d \psi_{S q}}{d \tau}+\omega_{m} \psi_{S d}
\end{aligned}
$$

where $u_{S d}, u_{S q}, \psi_{S d}, \psi_{S q}, i_{S d}, i_{S q}$ are the p.u. stator $d$ and $q$ axis voltage, flux linkage and current, $r_{S}$ is the p.u. stator resistance, $\omega_{m}$ is the p.u. rotor angular velocity and $\tau$ is the p.u. time.

The stator flux linkage viewed in a synchronous reference frame is given by (2).

$$
\begin{gathered}
\psi_{S d}=\ell_{S d} i_{S d}+\psi_{m} \\
\psi_{S q}=\ell_{S q} i_{S q}
\end{gathered}
$$


where $\ell_{S d}$ and $\ell_{S q}$ are the p.u. $d$ and $q$ axis inductances and $\psi_{m}=\left|\underline{\psi}_{m}\right|$ is the p.u. permanent magnet flux linkage.

The definiton of the $d$-q reference frame with respect to the stationary $(\alpha, \beta)$ reference frame and the stator flux linkage (2) (in case of $\underline{i}_{S}=j i_{S q}$ ) is illustrated in Fig. 2. $\gamma_{m}$ denotes the actual rotor angle.

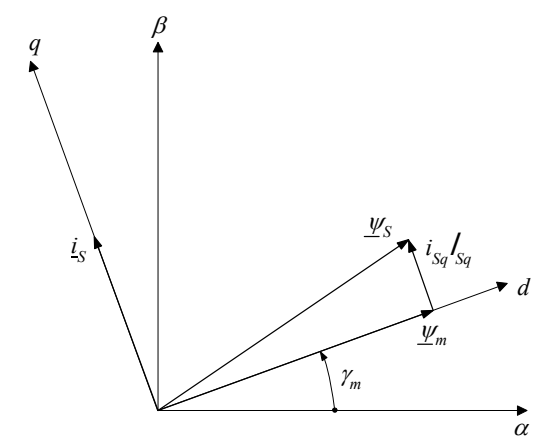

Figure 2: Definition of the synchronous $(d, q)$ reference frame and the stator flux linkage.

The p.u. torque in $d-q$ coordinates becomes

$$
t=\psi_{m} i_{S q}+\left(\ell_{S d}-\ell_{S q}\right) i_{S d} i_{S q}
$$

In order to complete the description of the PMSM the mechanical equation is necessary:

$$
\frac{d \omega_{m}}{d \tau}=\frac{1}{\tau_{m}}\left(t-t_{\text {lood }}\left(\omega_{m}\right)\right)
$$

where $\tau_{m}$ and $t_{L}$ are the p.u. moment of inertia and the p.u. load torque.

So far, no assumption was made regarding the inductances $\ell_{S d}$ and $\ell_{S q}$. The iron saturation can be easily considered by assuming the inductances dependent on the currents. Since cross coupling is taken into account each inductance is dependent on both, $i_{S d}$ and $i_{S q}$ [6]. Therefore, the general approach to consider saturation and cross coupling is:

$$
\begin{gathered}
\psi_{S d}=\ell_{S d}\left(i_{S d}, i_{S q}\right) \cdot i_{S d}+\psi_{m} \\
\psi_{S q}=\ell_{S q}\left(i_{S d}, i_{S q}\right) \cdot i_{S q}
\end{gathered}
$$

Applying (5) to (1) and using the derivation rules the voltage equations can be written as:

$$
\begin{gathered}
u_{S d}=r_{S} i_{S d}+\left(\ell_{S d}+a_{d d} i_{S d}\right) \frac{d i_{S d}}{d \tau}+i_{S d} a_{d q} \frac{d i_{S q}}{d \tau}-\omega_{m} \ell_{S q} i_{S q} \\
u_{S q}=r_{S} i_{S q}+\left(\ell_{S q}+a_{q q} i_{S q}\right) \frac{d i_{S q}}{d \tau}+i_{S q} a_{q d} \frac{d i_{S d}}{d \tau}+\omega_{m}\left(\ell_{S d} i_{S d}+\psi_{m}\right)
\end{gathered}
$$

where

$$
\begin{aligned}
& a_{d d}=\frac{\partial \ell_{S d}}{\partial i_{S d}}, a_{d q}=\frac{\partial \ell_{S d}}{\partial i_{S q}}, \\
& a_{q d}=\frac{\partial \ell_{S q}}{\partial i_{S d}}, a_{q q}=\frac{\partial \ell_{S q}}{\partial i_{S q}} .
\end{aligned}
$$

This leads to the following mathematical model of the PMSM: 


$$
\begin{gathered}
{\left[\begin{array}{c}
\frac{d}{d \tau} i_{S d} \\
\frac{d}{d \tau} i_{S q}
\end{array}\right]=\frac{1}{\Delta}\left[\begin{array}{cc}
\tilde{l}_{S q}+\tilde{a}_{q q} i_{S q} & -\tilde{a}_{d q} i_{S d} \\
-\tilde{a}_{q d} i_{S q} & \tilde{l}_{S d}+\tilde{a}_{d d} i_{S d}
\end{array}\right]\left[\begin{array}{c}
u_{S d}-\tilde{r}_{S} i_{S d}+\omega_{m} \tilde{r}_{S q} i_{S q} \\
u_{S q}-\tilde{r}_{S} i_{S q}-\omega_{m}\left(\tilde{l}_{s d} i_{S d}+\tilde{\psi}_{m}\right)
\end{array}\right]} \\
\Delta=\tilde{l}_{S d} \tilde{l}_{S q}+\tilde{l}_{S d} \tilde{a}_{q q} i_{S q}+l_{S q} \tilde{a}_{d d} i_{S d}+\left(\tilde{a}_{d d} \tilde{a}_{q q}-\tilde{a}_{d q} \tilde{a}_{q d}\right) i_{S d} i_{S q} \\
t=\tilde{\psi}_{m} i_{S q}+\left(\tilde{\ell}_{S d}-\tilde{\ell}_{S q}\right) i_{S d} i_{S q} \\
\frac{d \omega_{m}}{d \tau}=\frac{1}{\tau_{m}}\left(t-t_{\text {load }}\left(\omega_{m}\right)\right)
\end{gathered}
$$

where $\sim$ indicates the parameters which are changeable during the operation of the PMSM.

This modell has been used for the simulation. The parameters $r_{S}$ and $\psi_{m}$ have to be accepted as temperature dependent and therefore also as changeable during the operation of the PMSM.

\section{ROBUST BACKSTEPPING CONTROL FOR A PMSM WITH NONLINEAR LOAD}

The conventional control scheme for a PMSM consists of a decoupling network and a linear controller. Since we want to examine cases in which the nonlinear nature of the control task has to be taken into account, we focus our attention on a PMSM coupled with a nonlinear load and we assume that the exact characteristic is unknown (uncertain) but bounded by some lower and upper characteristics:

$$
\begin{gathered}
t_{\text {load , upper }}\left(\omega_{m}\right)=\omega_{m}{ }^{2}-0.4 \omega_{m}+0.09 \\
t_{\text {load, lower }}\left(\omega_{m}\right)=0
\end{gathered}
$$

Fig. 3 shows the bounding characteristics and some characteristic curve described by these bounds.

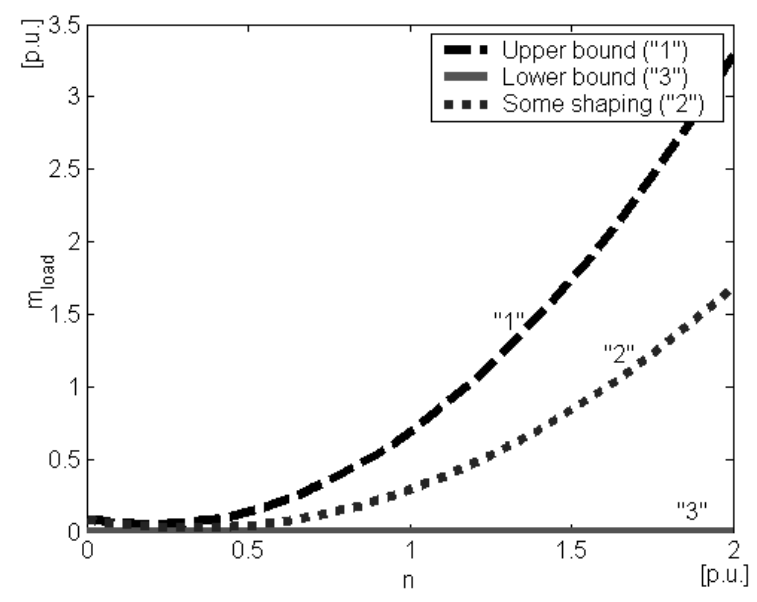

Figure 3: Speed torque characteristics with lower and upper border.

These characteristics could describe a rotary pump or a ventilator.

Backstepping is a recursive design procedure. In each step of the iteration we get a more complicated virtual control law. During the last step we get the control law itself. Cause of lack of space we omit the basics of backstepping and focus our attention to the specialities of this application. We refer to [1], [2] and [12] for more details. The crosscoupling effect has been omitted during this approach but considered during simulation. In order to design the backstepping controller we choose different coordinates [12]. This model of the PMSM is a 
square multivariable system (inputs: $u_{S d}, u_{S q}$, outputs: $\left.\omega_{m}, i_{S d}\right) . i_{S d}$ should be driven to zero in order to avoid demagnetization. We define the first set of the so called "backstepping controller coordinates" as

$$
\begin{aligned}
& z_{11}=\omega_{m}-\omega_{m, r e f}(\tau), \\
& z_{21}=i_{S d}
\end{aligned}
$$

where $z_{11}$ is the speed tracking error. In the ideal case both coordinates $z_{11}, z_{21}$ should equal 0 . Then the speed of the PMSM would equal the reference speed and the stator current in the ddirection would vanish.

The first derivatives regarding time using (8) are:

$$
\begin{aligned}
& \dot{z}_{11}=\frac{\psi_{m} i_{S q}+\left(\ell_{S d}-\ell_{S q}\right) i_{S d} i_{S q}-t_{\text {load }}\left(\omega_{m}\right)}{\tau_{m}}-\frac{d \omega_{m, r e f}(\tau)}{d t} \\
& \dot{z}_{21}=\frac{u_{S d}-r_{S} i_{S d}+\omega_{m} \ell_{S q} i_{S q}}{\ell_{S d}}
\end{aligned}
$$

We assume as next step the following virtual inputs [12]:

$$
\begin{gathered}
v_{1}=\frac{\psi_{m} i_{S q}+\left(\ell_{S d}-\ell_{S q}\right) i_{S d} i_{S q}}{\tau_{m}} \\
v_{2}=u_{s d}
\end{gathered}
$$

The second set of the controller coordinates is defined as (since in the time derivative of $z_{21}$ we found already the input $u_{S d}$, there is no $z_{22}$ ):

$$
z_{12}=v_{1}-\alpha_{11}
$$

where $\alpha_{11}$ is the virtual control law of the first step of the backstepping design procedure. Inserting (3), (4) into (2) we get:

$$
\dot{z}_{11}=z_{12}+\alpha_{11}-\frac{t_{\text {real }}\left(\omega_{m}\right)}{\tau_{m}}-\frac{d \omega_{m, r e f}(\tau)}{d \tau}
$$

A reasonable choice for the first virtual control law is:

$$
\alpha_{11}=-c_{11} z_{11}-d_{11} \int_{0}^{t} z_{11} d \tau-\tanh \left(\sigma_{1} z_{11}\right) \frac{t_{\text {load }, \text {,pper }}\left(\omega_{m}\right)}{\tau_{m}}+\frac{d \omega_{m, \text { ref }}(\tau)}{d \tau}
$$

where $c_{11}$ and $d_{11}$ are positive controller parameters.

Validity of this choice of virtual control law can be demonstrated by a Lyapunov argument. The term $\tanh \left(\sigma_{1} z_{11}\right) \frac{t_{\text {load, upper }}\left(\omega_{m}\right)}{\tau_{m}}$ is a robust extension and added to handle the uncertain nonlinear load, $\tanh \left(\sigma_{1} z_{11}\right)$ is used instead of $\operatorname{sign}\left(z_{11}\right)$ in order to counteract a too aggressive behaviour of the controller, $\sigma_{1}$ is a form parameter determined by simulation runs. We get as consequence of this choice ultimate bounded stability instead of global asymptotic stability of the origin in $z$-coordinates. By using (14) and (15) the dynamics of $z_{11}$ therefore becomes: 


$$
\dot{z}_{11}=-c_{11} z_{11}-d_{11} \int_{0}^{t} z_{11} d \tau+z_{12}-\tanh \left(\sigma_{1} z_{11}\right) \frac{t_{\text {load }, \text { upper }}\left(\omega_{m}\right)}{\tau_{m}}-\frac{t_{\text {load }}\left(\omega_{m}\right)}{\tau_{m}}
$$

Since backstepping is a recursive design procedure the next steps are intelligible, we compute the time-derivative of (6).

$$
\dot{z}_{12}=\dot{v}_{1}-\dot{\alpha}_{1}=\frac{d}{d \tau}\left(\frac{\psi_{m} i_{S_{q}}+\left(\ell_{S_{d}}-\ell_{S_{q}}\right) i_{S d} \dot{i}_{S q}}{\tau_{m}}\right)-\frac{d}{d \tau}\left(-c_{11} z_{11}-d_{11} \int_{0}^{t} z_{11} d \tau-\tanh \left(\sigma_{1} z_{11}\right) \frac{t_{\text {load, }, \text { ppor }}\left(\omega_{m}\right)}{\tau_{m}}+\frac{d \omega_{m, r e f}(\tau)}{d \tau}\right)
$$

An analytical computation of the time derivative of all terms in (17) is possible. Since the time derivative contains the input variables, this is the last step of the procedure. Again we determine a desirable dynamics of the backstepping controller coordinate:

$$
\dot{z}_{12}=-c_{12} z_{12}-z_{11}+\Delta_{\text {unc }}-\tanh \left(\sigma_{2} z_{12}\right) \Delta_{\text {upperbound }}
$$

where $c_{11}$ and $\sigma_{2}$ are positive controller parameters and in $\Delta_{\text {unc }}$ are collected all uncertain parts of (17). $\Delta_{\text {upper bound }}$ is an expression that dominates $\Delta_{\text {unc }}$ in the sense of $\Delta_{\text {upper bound }}>\left|\Delta_{\text {unc }}\right|$.

If we evaluate (17),(18) and (11) we get a matrix inequality for $u_{S d}, u_{S q}$ of the kind:

$$
\mathbf{A}\left[\begin{array}{l}
u_{S d} \\
u_{S q}
\end{array}\right]=\left[\begin{array}{l}
f_{1}\left(z_{11}, z_{12}, z_{21}, \ldots\right) \\
f_{2}\left(z_{11}, z_{12}, z_{21}, \ldots\right)
\end{array}\right]
$$

with

$$
\mathbf{A}=\left[\begin{array}{cc}
\frac{i_{S q}\left(l_{S d}-l_{S q}\right)}{l_{S d} \tau_{m}} & \frac{\frac{\psi_{m}}{l_{S q}}+\frac{i_{S d}\left(l_{S d}-l_{S q}\right)}{l_{S q}}}{\tau_{m}} \\
\frac{1}{l_{S d}} & 0
\end{array}\right]
$$

In a reasonable operating region $\operatorname{det}(\mathbf{A}) \neq 0$ we can compute directly from (19) the stator voltage applied by the controller.

To complete the discussion we discuss stability of the approach, but we limit the discussion to the case of a certain load with no robust extension in the backstepping control law. In order to show stability the following Lyapunov function is introduced:

$$
V=\frac{1}{2}\left(z_{11}^{2}+z_{12}^{2}+z_{21}^{2}+d_{11} \gamma_{1}^{2}+d_{21} \gamma_{2}^{2}\right)
$$

where $\gamma_{1}=\int_{0}^{t} z_{11} d \tau$ and $\gamma_{2}=\int_{0}^{t} z_{21} d \tau$.

We obtain a linear control system dynamics in the case of exact backstepping as follows:

$$
\left[\begin{array}{c}
\dot{z}_{11} \\
\dot{z}_{12} \\
\dot{z}_{21} \\
\dot{\gamma}_{1} \\
\dot{\gamma}_{2}
\end{array}\right]=\left[\begin{array}{ccccc}
-c_{11} & 1 & 0 & -d_{11} & 0 \\
-1 & -c_{12} & 0 & 0 & 0 \\
0 & 0 & -c_{21} & 0 & -d_{21} \\
1 & 0 & 0 & 0 & 0 \\
0 & 0 & 1 & 0 & 0
\end{array}\right]\left[\begin{array}{c}
z_{11} \\
z_{12} \\
z_{21} \\
\gamma_{1} \\
\gamma_{2}
\end{array}\right]
$$


The system described by this state equation is stable as long as all the controller parameters are positive. In the case of robust backstepping the entire system dynamics is no longer linear and stability in this case can also be checked by the use of a standard Lyapunov argument [2].

\section{IMPROVING CONTROL LAW DYNAMICS BY LYAPUNOV FUNCTION FORMING}

Since we want to have a smooth and non aggressive control law, we apply a method formally introduced by Freeman and Kokotovic [13]. Square-type Lyapunov functions are very common cause of their special suitability for treatment. A slight alteration incorporates also the aspect of wellshapedness of the control law. The idea behind this "soft" approach is simple and tricky at the same time.

In order to establish Lyapunov functions which reduce the high dynamics of the control law we split the state space (in backstepping controller coordinates $z_{11}, z_{12}, z_{21}, z_{22}$ ) in different regions which are characterized by different control laws. In Fig. 4 a resonable splitting for two dimensions $\left(\gamma_{1}=0, \gamma_{2}=0, z_{21}=0\right)$ is shown. The border functions are chosen as:

$$
\begin{aligned}
& r_{1, O}\left(z_{11}\right)= \begin{cases}b\left(z_{11}-a\right)^{2}+a & z_{11}<-a \\
a & z_{11} \geq-a\end{cases} \\
& r_{1, U}\left(z_{11}\right)= \begin{cases}-b\left(z_{11}-a\right)^{2}-a & z_{11} \geq a \\
-a & z_{11}<a\end{cases}
\end{aligned}
$$

$b, a$ are parameters of the algorithm which have to be chosen appropriately. We found good values by simulation runs.

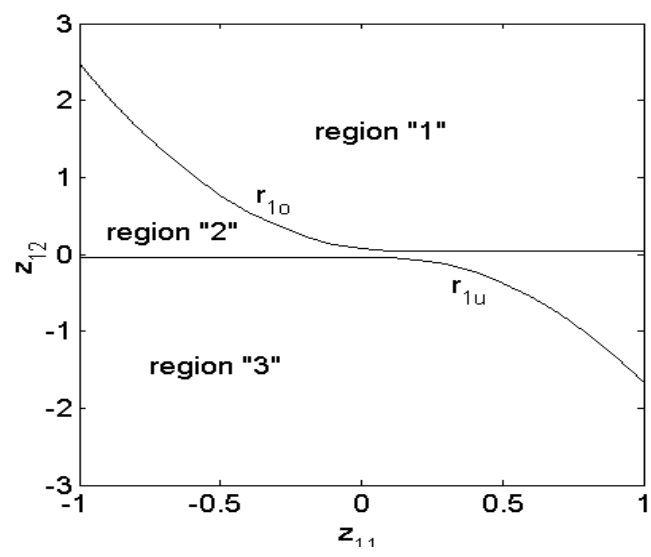

Figure 4: Splitting the state space into different regions to improve control law dynamics.

Instead of using the normal square-type Lyapunov function:

$$
V=\frac{1}{2} \mathbf{z}^{\mathrm{T}} \mathbf{z}=\frac{1}{2}\left(z_{11}^{2}+z_{12}^{2}+z_{21}^{2}+d_{11} \gamma_{1}^{2}+d_{21} \gamma_{2}^{2}\right)
$$

we use a slightly different Lyapunov function which depends on the region:

$$
V=\left\{\begin{array}{cc}
\frac{1}{2}\left(z_{11}^{2}+\left(z_{12}-r_{10}\left(z_{11}\right)\right)^{2}+z_{21}^{2}+d_{11} \gamma_{1}^{2}+d_{21} \gamma_{2}^{2}\right) & \left(z_{11}, z_{12}\right) \in \text { region"1" } \\
\frac{1}{2}\left(z_{11}^{2}+z_{12}^{2}+z_{21}^{2}+z_{22}^{2}+d_{11} \gamma_{1}^{2}+d_{21} \gamma_{2}^{2}\right) & \left(z_{11}, z_{12}\right) \in \text { region"2" } \\
\frac{1}{2}\left(z_{11}^{2}+\left(z_{12}-r_{1 U}\left(z_{11}\right)\right)^{2}+z_{21}^{2}+d_{11} \gamma_{1}^{2}+d_{21} \gamma_{2}^{2}\right) & \left(z_{11}, z_{12}\right) \in \text { region"3" }
\end{array}\right.
$$


What does this mean geometrically? The interpretation of the Lyapunovfunction is equivocal. One interpreation is to consider the Lyapunov function as a penalty function. By choosing the new Lyapunov function (25) the penalty function is no longer the square of the distance to the origin in the $\left(z_{11}, z_{12}, z_{21}, \gamma_{1}, \gamma_{2}\right)$ state space, but the square of the distance to some point on the $z_{12}$-axes. This interrelation (in two dimensions) is shown in Fig. 5 for two different states.

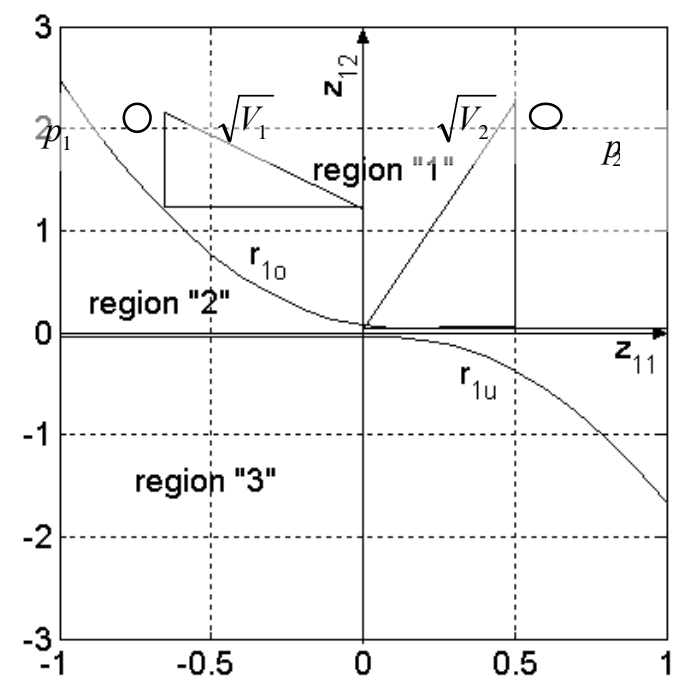

Figure 5: Interpretation of the Lyapunovfunction as the square of the distance to some point in the state space.

This special forming of the Lyapunov function leads to a different control law. In each region of the state space $\left(z_{11}, z_{12}, z_{21}, \gamma_{1}, \gamma_{2}\right)$ we get a different control law. These single control laws have a continuous transition at the region-borders.

The control law design according to this approach with a slightly changed Lyapunov function (25) is quite straightforward, just some few minor changes as opposed to the classical approach (10)-(20) have to be implemented.

\section{SIMULATIONS AND RESULTS}

In order to simulate the control concept an appropriate simulation environment for MATLAB/Simulink has been established. The PMSM and two control algorithms (conventional control with a decoupling network and soft robust backstepping control) have been implemented as s-functions. The soft robust backstepping control algorithm has been computed with MAPLE and then converted to an s-function (Conversions->Matlab). The soft robust backstepping algorithm has been implemented in the function mdloutputs of a standard s-function. During simulation the cross coupling effect of the PMSM is taken into account. The power electronics is modelled with a time delay $T_{D}$ for the $\mathrm{d}$ - and q-component of the stator voltage respectively:

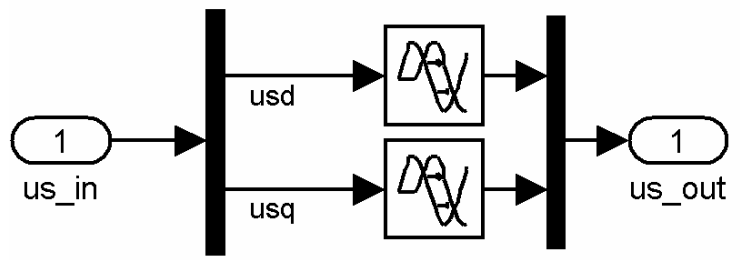

Figure 6: Modelling of the power electronics. 
The simulation model contains a continuous time part (the PMSM) and a discrete time part (the controller).

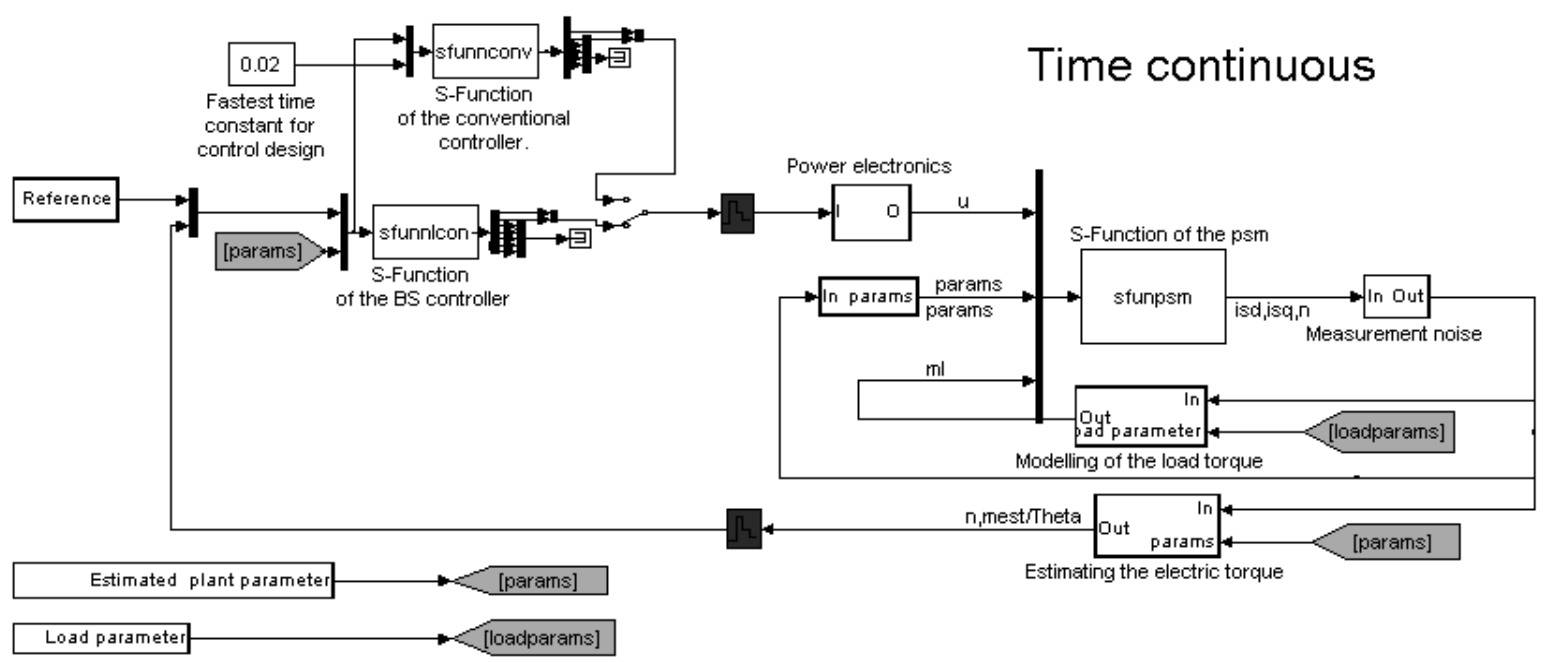

Time discrete

Figure 7: Simulation model of the introduced control concept.

The parameters for the simulation and the parameters of the PMSM are the following:

\begin{tabular}{|l|l|}
\hline Sample time & $T_{A}=5 \mathrm{~ms}$ \\
\hline Stator resistance & $r_{S}=0.03$ \\
\hline Permanent flux & $\Psi_{m}=0.9$ \\
\hline Time delay of the power electronics & $T_{D}=20 \mathrm{~ms}$ \\
\hline
\end{tabular}

Following values of the parameters of the crosscoupling effect have been used for the simulation runs:

\begin{tabular}{|l|l|}
\hline Stator inductivity d-direction & $l_{S d}=-0.05 i_{S d}+0.02 i_{S q}+0.37$ \\
\hline Stator inductivity q-direction & $l_{S q}=-0.02 i_{S d}-0.02 i_{S q}+0.41$ \\
\hline Cross-coupling coefficient d->d & $a_{d d}=-0.05$ \\
\hline Cross-coupling coefficient d-> q & $a_{d q}=0.02$ \\
\hline Cross-coupling coefficient q->d & $a_{q d}=-0.02$ \\
\hline Cross-coupling coefficient q->q & $a_{q q}=-0.02$ \\
\hline
\end{tabular}

The controller parameters are as follows:

\begin{tabular}{|c|}
\hline$c_{11}=10$ \\
\hline$c_{12}=10$ \\
\hline$c_{21}=20$ \\
\hline$d_{11}=200$ \\
\hline$d_{21}=20$ \\
\hline$\sigma_{1}=1$ \\
\hline$\sigma_{2}=1$ \\
\hline$a=0.05$ \\
\hline$b=1$ \\
\hline
\end{tabular}



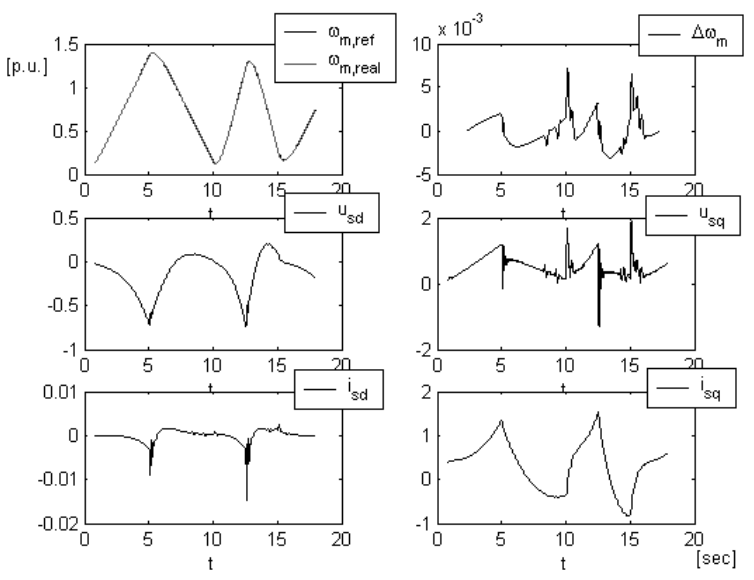

Figure 8: Soft robust backstepping control with a load between the lower and upper bounding load characteristic.

From Fig. 8 and 9 can be seen that the tracking goal is achieved quite well. The tracking error is less than $7 \%$. This is achieved with different loads. In all simulations it is assumed that some parameters differ slightly from their assumed values:

\begin{tabular}{|l|l|}
\hline Stator resistance & $r_{S, \text { real }}=0.035$ \\
\hline Permanent flux & $\Psi_{m, \text { real }}=0.7$ \\
\hline
\end{tabular}
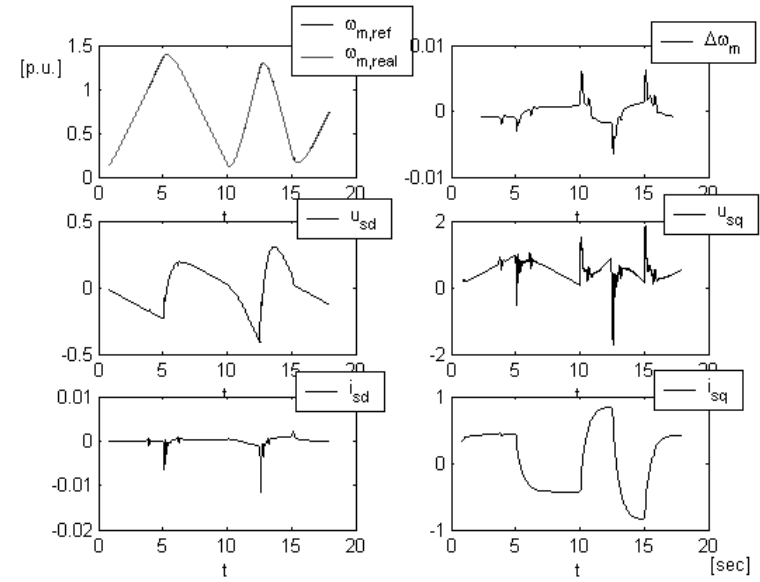
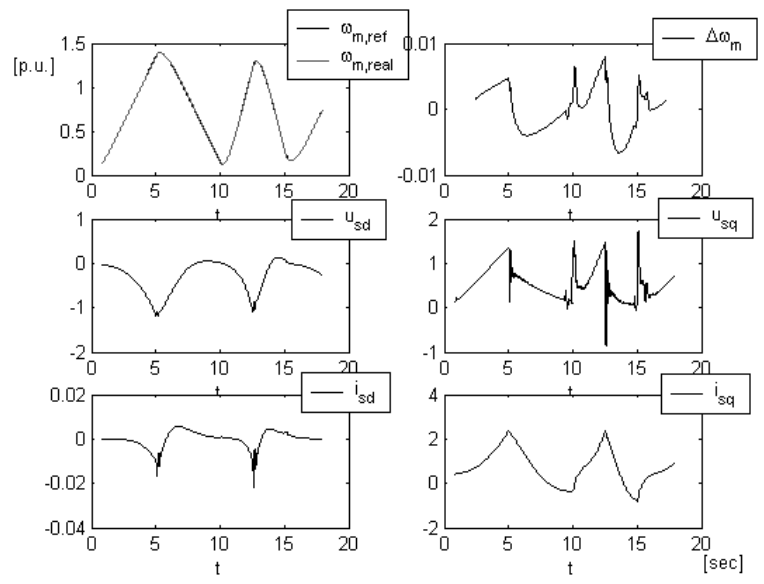

Figure 9: Soft robust backstepping control with the lower bounding characteristic (left) and the upper bounding charcteristic (right) as load.

In Fig. 10 a conventional linear controller with decoupling network is compared to the soft robust backstepping approach. We notice that the tracking performance of soft robust backstepping approach is better than the conventional controller although this advantage has a more aggressive control law as consequence. 

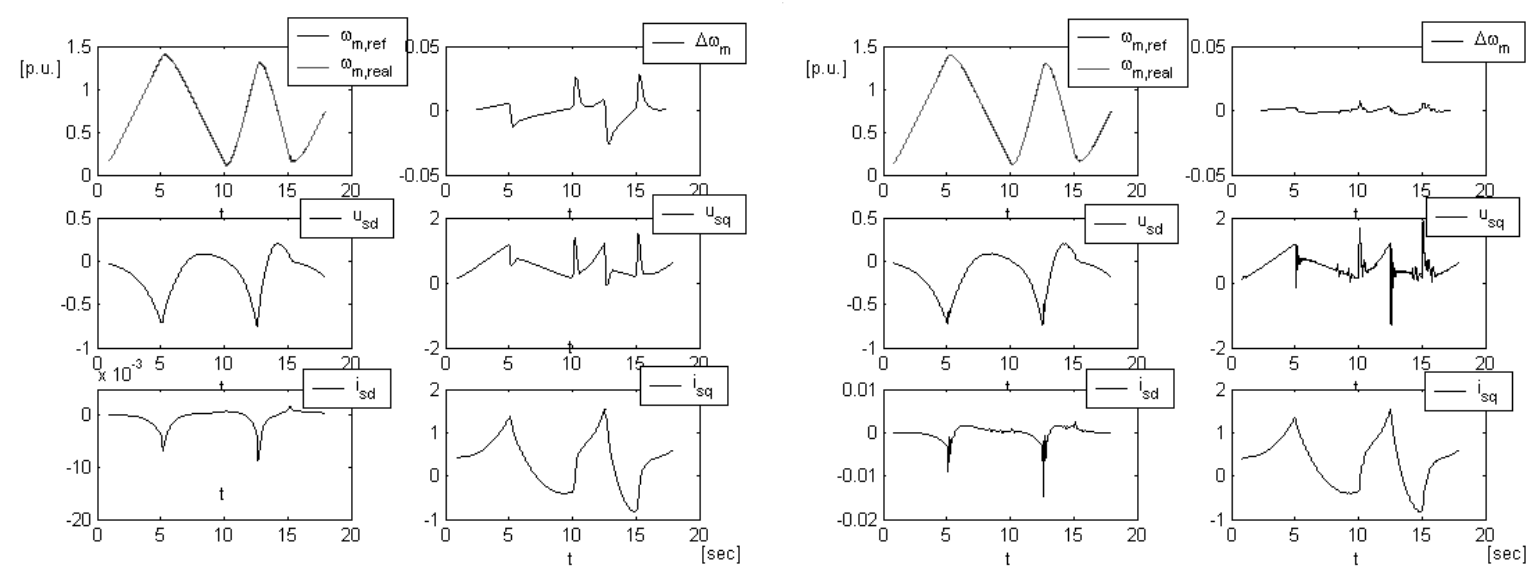

Figure 10: Conventional (left side) and soft robust backstepping control (right side) with a load between the bounding characteristics.

\section{CONCLUSIONS}

Heavy nonlinear load affecting a PMSM force the control engineer to reconsider the control design process. Backstepping is an especially suitable design technique in this case. An approach which introduces a new idea to the classic square-type Lyapunov function for backstepping control design has been adapted to the pecularities of the PMSM and its describing equations. The approach leads to high precision in following a reference trajectory with reasonable control law amplitude (control authority) and dynamics. Since the approach is a robust control design technique no adaptation of parameters takes places. The determination of the optimal controller parameters is a difficult task, good parameter constellations have been found by simulation runs. The implementational effort for the introduced controller is high compared to conventional control solutions for the PMSM (with a decoupling network). For slight nonlinearities conventional linear control of the PMSM seems to be the the better approach, but for heavy nonlinear load the applied method seems to be quite appropriate.

Next step would be the implementation on a real system. The validity and some advantages of the method have been shown in principle by the use of simulation. So the authors consider the paper as a step towards an applicable control method for a PMSM affected by a heavy nonlinear load with high demands on precision.

\section{ACKNOWLEDGEMENT}

The authors thank Prof. Weinmann for discussion.

\section{REFERENCES}

[1] Sepulchre, R.; Jankovic, M.; Kokotovic, P. V. (1997). Constructive Nonlinear Control, Springer, Vienna, New York

[2] Viehweider, A. (2003). Robustes Backstepping für den Nichtlinearen Entwurf, Tagungsband 13. Steirisches Seminar über Regelungstechnik und Prozessautomatisierung, Schloss Retzhof, 184205

[3] Carroll, J. J.; Dawson, D. M. (1995). Integrator backstepping techniques for the tracking control of permanent magnet brush DC motors, IEEE Transaction on Industrial Application, Vol. 31, No. 2, 248-255 
[4] Yang, Z.; Minashima, M. (2001). Robust Nonlinear Control of a Feedback Linearizable VoltageControlled Magnetic Lévitation System, T.IEE Japan, Vol. 121-C, No. 7, 1203-1211

[5] Tan, Y.; Chang, J.; Hu, J.; Tan, H. (2000). Advanced Nonlinear Control Strategy for Motion Control Systems, Report Rockwell Science Center, Thousand Oaks, 117-121

[6] Zhou, J.; Wang, Y. (2002). Adaptive backstepping speed controller design for a permanent magnet synchrous motor, IEE Proc.-Electr. Power Appl., Vol. 149, No. 2, 165-172

[7] Der-Fa, Ch.; Tian-Hua, L.; Che-Kai, H. (2002). Adaptive backstepping controller design for a matrix converter based PMSM drive system, Proceedings IEEE ICIT'02, Bangkok, Thailand, 258-263

[8] Xu, Jian-Xin; Lee, Tong Heng (1998). On adaptive robust backstepping control schemes suitable for PM synchronous motors, Int. J. Control, Vol. 70, No. 6, 893-920

[9] In-Cheol, B.; Kyeong-Hwa, Kim (1999). DSP-Based Robust Nonlinear Speed Control of PM Synchronous Motor, Electric Machines and Power Systems, Vol. 27, 481-499

[10] Glasl, M.; Wittmann, B. (2003). Feldorientierte Regelung der PermanentmagnetSynchronmaschine auf Basis des Drehzahlistwertes mit Winkelfehlerkorrektur, $e \& i$ elektrotechnik und informationstechnik, Heft 1, 37-41

[11] Kilthau, A.; Pacas, J. M. (2001). Parameter-Measurement and Control of the Synchronous Reluctance Machine including Cross Saturation, Conf. record of IEEE-IAS Annual Meeting, Vol. 4, 2302-2309

[12] Viehweider, A. (2004). A Design and Optimization Method for Robust Backstepping Control with Integral Action, Conf. proceeding. Cybernetics and Systems 2004 Vienna, Vol. 2, 23022309

[13] Freeman, R. A.; Kokotovic, P. V. (1993). Design of `Softer` Robust Nonlinear control laws, Automatica, Vol. 29, 1425-1437 